\title{
Creating a Database of Spatial Structure Characteristics of Surface Waves in the Russian Black Sea Shelf Area
}

\author{
Victor V. Zamshin, Alexander G. Beloborodov, Alexandra Sh. Zamshina \\ AEROCOSMOS Research Institute for Aerospace Monitoring, Moscow, Russia \\ office@aerocosmos.info
}

\begin{abstract}
A relational database containing surface wave spatial structure characteristics has been created. This database allows us to collect, systematize, store, and study wave spectra in the framework of monitoring of anthropogenic impacts on marine water areas of the Russian Black Sea shelf area. Spatial spectra of marine surface measured using satellite imagery and by contact methods, as well as marine surface slopes and elevations retrieved using satellite imagery are the source data for this database. The generated array of data is stored in the network storage. Necessary information can be requested using the developed client application through SQL queries, as well as through typical queries as app interface functions. An example of thematic use of this database to analyze information features of spatial spectra of the water surface optical satellite image is given here.
\end{abstract}

Keywords: databases, remote sensing, satellite imagery, satellite monitoring, marine water areas, wave spatial structure, wave spectra, anthropogenic impacts 


\title{
ФОРМИРОВАНИЕ БАЗЫ ДАННЫХ ХАРАКТЕРИСТИК ПРОСТРАНСТВЕННОЙ СТРУКТУРЫ ПОВЕРХНОСТНОГО ВОЛНЕНИЯ НА РОССИЙСКОМ ШЕЛЬФЕ ЧЁРНОГО МОРЯ
}

\author{
В.В. Замиин, А.Г. Белобородов, А.Ш. Замиина \\ Научно-исследовательский институт аэрокосмического мониторинга "АЭРОКОСМОС", Москва, \\ Россия \\ office@aerocosmos.info
}

Сформирована реляционная база данных характеристик пространственной структуры поверхностного волнения, которая позволяет собирать, систематизировать, хранить и изучать спектры волнения для решения задачи мониторинга антропогенных воздействий на морские акватории российского шельфа Чёрного моря. В качестве исходной информации выступают пространственные спектры космических изображений морской поверхности, измеренные контактными методами спектры морского волнения, а также восстановленные по космическим изображениям спектры уклонов и возвышений морской поверхности. Сформированный массив данных хранится на сетевом хранилище. Необходимая пользователю информация может быть запрошена у базы данных через разработанное клиентское приложение путём выполнения SQL-запросов, а также с помощью интегрированных в приложение интерфейсных функций типовых запросов. Приведён пример тематического использования базы данных для анализа информативных признаков массива пространственных спектров космических оптических изображений водной поверхности.

Ключевые слова: базы данных, дистанционное зондирование Земли, космические изображения, спутниковый мониторинг, морские акватории, пространственная структура волнения, спектры волнения, антропогенные воздействия.

\section{Введение}

Для систематизации и хранения больших объемов информации, получаемой при проведении дистанционного зондирования Земли из космоса, целесообразно использование баз данных (БД), т.е. автоматизированных систем, позволяющих хранить космические изображения, результаты их обработки и сопроводительную информацию в удобной для дальнейшей обработки форме. Создание таких систем актуально, в том числе, и для решения задач исследования прибрежных акваторий в интересах оценки уровня антропогенных воздействий и выработки обоснованных управленческих решений, снижающих риск негативных экологических и экономических последствий от загрязнения водной среды [1-5].

В рамках выполнения научных исследований, посвящённых мониторингу пространственной структуры морского волнения в интересах оценки антропогенных воздействий на акватории российского шельфа Чёрного моря, требовалось разработать БД, позволяющую собирать, систематизировать, хранить и изучать данные следующих типов: двумерные спектры фрагментов космических изображений морской поверхности, полученные на основе алгоритма быстрого преобразования Фурье (БПФ); спектры морского волнения, полученные контактными методами (волнографы, волновые буи и пр.); спектры уклонов и возвышений морской 
поверхности, восстановленные по двумерным спектрам космических изображений [2,6-9]. В рамках решения этой задачи была разработана «База данных характеристик пространственной структуры поверхностного волнения на российском шельфе Чёрного моря» (БДХПВ).

\section{Особенности базы данных}

В созданной БДХПВ используется реляционная модель данных, которая подразумевает, что объекты реального мира и взаимосвязи между ними представляются в виде совокупности таблиц, называемых отношениями $[1,4]$. Каждая таблица представляет объект и содержит в себе множество строк и столбцов. В реляционной БД каждая таблица должна иметь первичный ключ - поле или совокупность полей, которые единственным образом идентифицируют каждую строку в таблице. При создании информационных систем совокупность отношений таблиц позволяет хранить данные об объектах предметной области и моделировать связи между ними [4].

Разработанная на основе реляционной модели БДХПВ (рис.1) реализует объективную форму представления и организации тематических данных, обеспечивает их целостность, позволяет организовать хранение данных с учетом специфики предметной области, характеризующейся, с одной стороны, необходимостью хранения большого количества спектров и их характеристик, а с другой стороны, необходимостью предоставления исследователям оперативного доступа к обрабатываем данным и их информативным признакам в удобной форме.

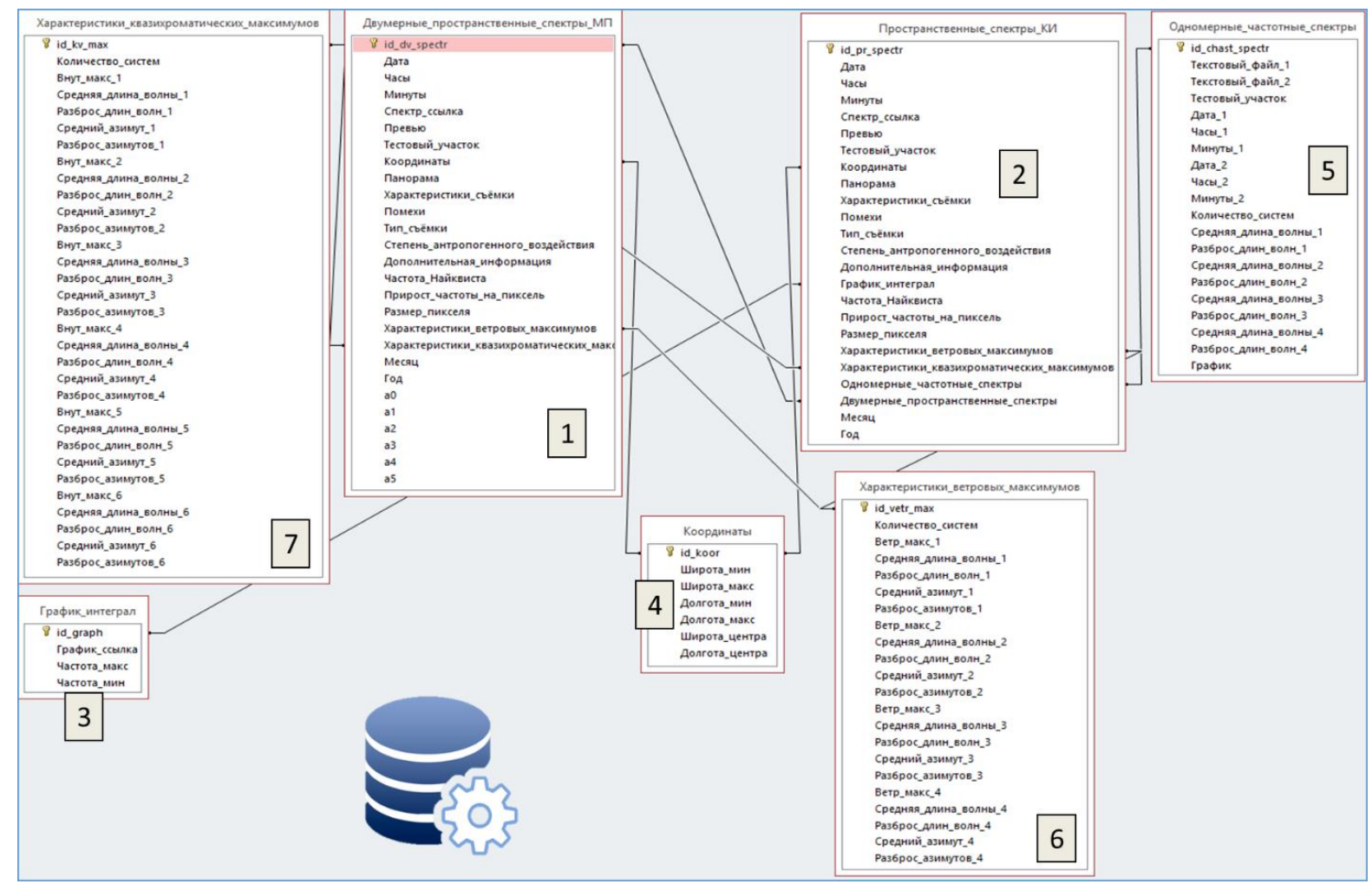

Рис. 1. Схема базы данных характеристик пространственной структуры поверхностного волнения на российском шельфе Чёрного моря

Как видно из рис.1, в БДХПВ используется 7 типов таблиц. Таблица 1 («Двумерные пространственные спектры морской поверхности») содержит данные о восстановленных по космическим изображениям двумерных пространственных спектрах уклонов и возвышений морской поверхности, а также ссылки на файлы спектров и их превью. Таблица 2 («Пространственные спектры космических изображений») содержит данные о пространственных 
спектрах фрагментов космических изображений, полученных на основе БПФ, а также ссылки на файлы спектров и их превью. Таблица 3 («Интегрированный спектр») содержит данные об угловом распределении энергии в пространственных спектрах. Таблица 4 («Координаты») содержит сведения о географическом положении спектров. Таблица 5 («Одномерные частотные спектры») содержит сведения о спектрах морской поверхности, измеренных «in-situ». Таблица 6 («Характеристики ветровых максимумов») содержит сведения о характеристиках ветровых максимумов, зафиксированных в каком-либо из спектров. Таблица 7 («Характеристики квазимонохроматических максимумов») содержит сведения о характеристиках «квазимонохроматических» максимумов, выявленных в каком-либо из спектров, обусловленных проявлениями на морской поверхности внутренних волн, генерируемых, в том числе, в результате воздействий глубинных стоков, поступающих в водную среду из коллекторов сбросовых устройств [2,5,7,8].

Клиентское приложение, разработанное при создании БДХПВ, обеспечивает возможность внесения, просмотра и редактирования данных авторизованными пользователями. Заложенные в приложение алгоритмы позволяют проверять корректность внесенных данных, быстро редактировать сохранённую информацию, предоставлять отчёты об ошибках с рекомендациями по их устранению и др. Приложение обладает независимым от стороннего программного обеспечения визуализатором изображений, а также рядом специализированных функций, облегчающих работу со спектрами (рис.2).

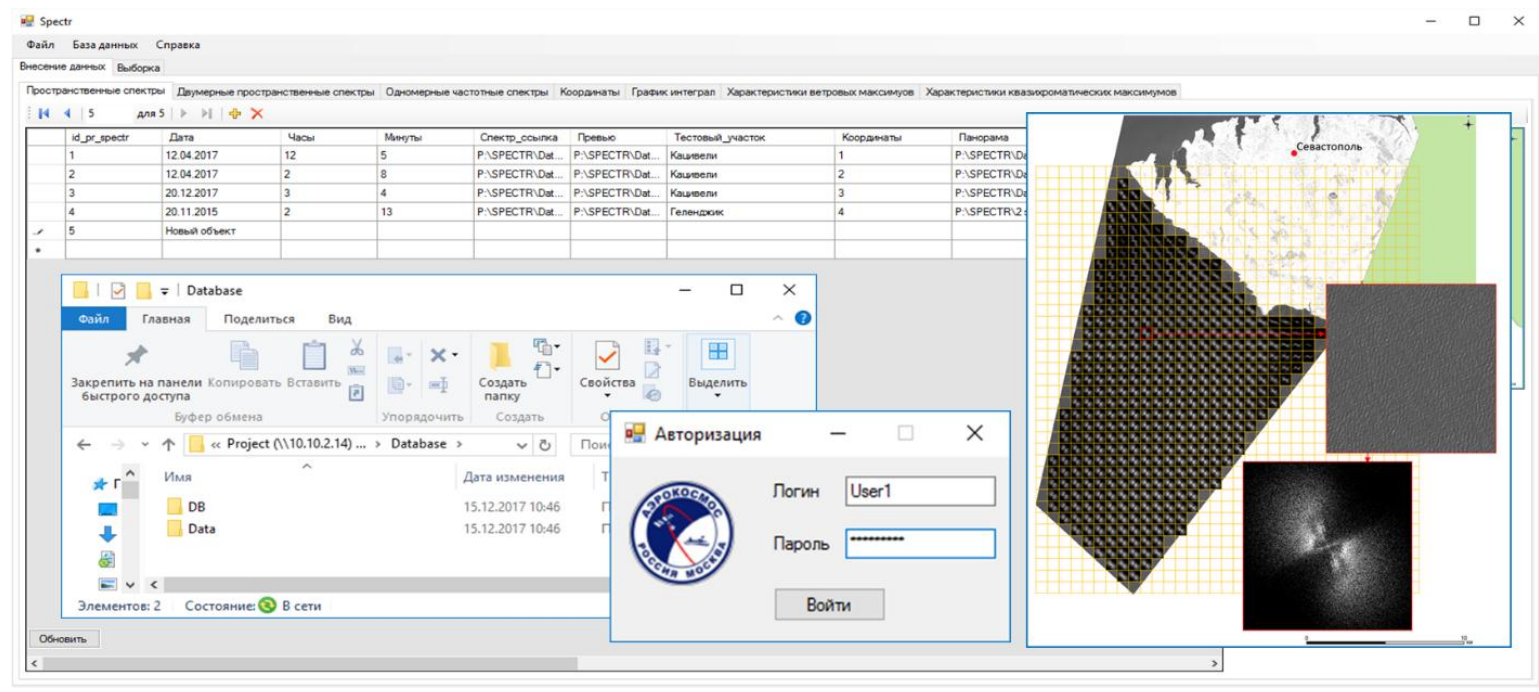

Рис. 2. Примеры интерфейсов клиентского приложения базы данных характеристик пространственной структуры поверхностного волнения на российском шельфе Чёрного моря

Для повышения функциональности БДХПВ было организовано сетевое хранилище с системой резервирования данных на основе RAID-массива, обеспечивающее надёжность хранения данных, бесперебойную работу, лёгкость доступа для большого числа пользователей, масштабируемость. Прямым доступом к файловой системе, а также возможностями контроля ключевых функций Бд обладает администратор, пользователи имеют ограниченные права и работают с данными через клиентское приложение. На данном этапе работы пользователям БДХПВ являются сотрудники организации-разработчика.

\section{Пример использования базы данных в интересах мониторинга пространственной структуры поверхностного волнения}

Как уже было показано, одним из видов информации, хранимой в БДХПВ, являются двумерные спектры фрагментов космических изображений морской поверхности. На рис. 2 справа приведён пример фрагмента космического оптического изображения и соответствующего ему 
пространственного спектра, а также пример схемы регулярной сетки, на которой проводилась обработка изображения с получением спектров. Такие спектры характеризуют структуру поверхностного волнения. Например, на основании исследования характеристики спектральных максимумов можно получать информацию о количестве систем, пространственных периодах, направлениях распространения и других параметрах поверхностных волн в исследуемой акватории [5,6,8]. Разработанная БДХПВ позволяет системно хранить, быстро запрашивать и исследовать такие характеристики в многопользовательском режиме.

На рис.3 приведены примеры результатов обработки выгруженных из БДХПВ характеристик спектральных максимумов, выделенных на основании интерактивного анализа пространственных спектров 126-и фрагментов космического оптического панхроматического изображения морской поверхности, полученного со спутника Ресурс-П 16.04.2017 в районе п. Кацивели (Крым).

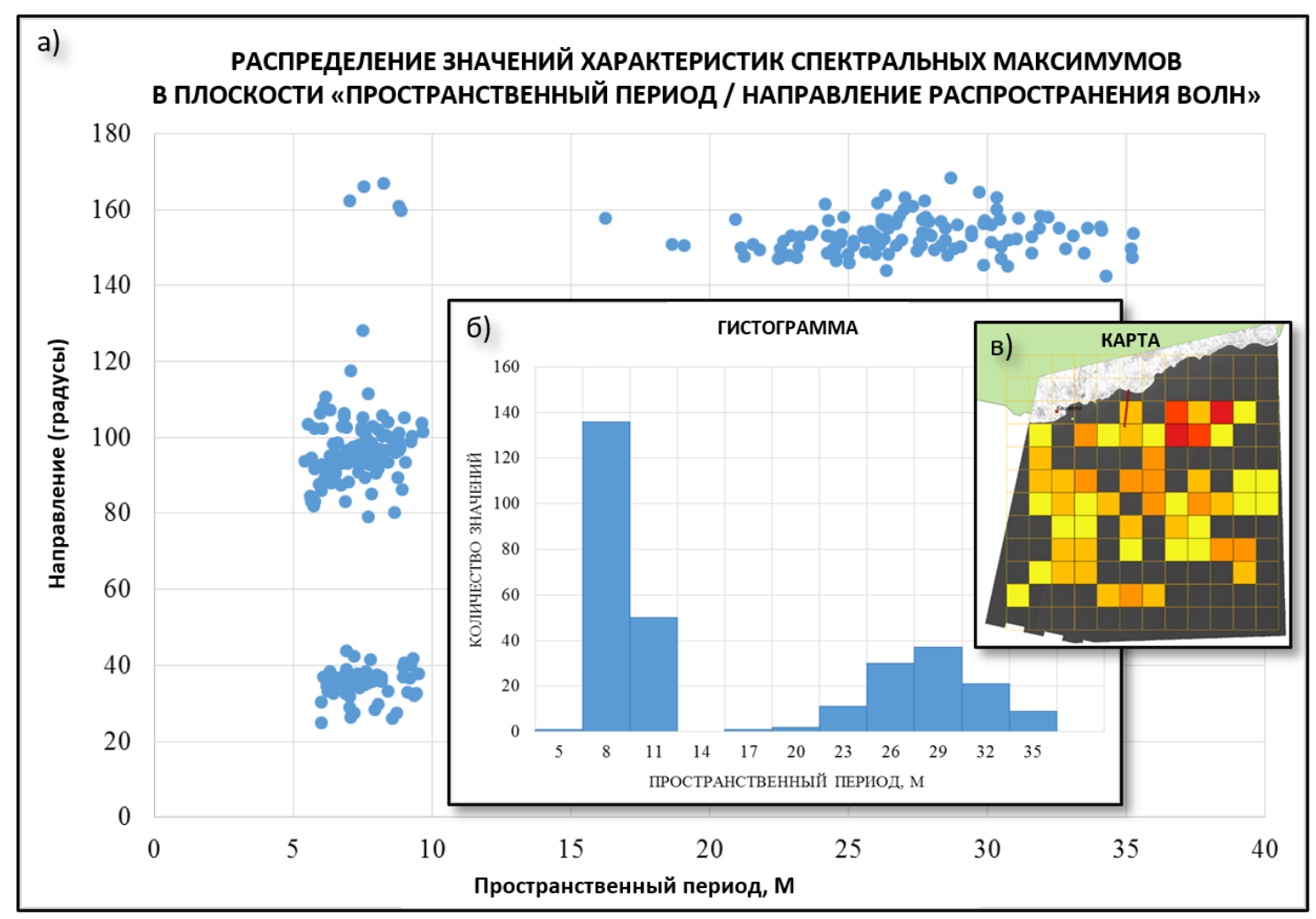

Рис. 3. Примеры результатов обработки выгруженных из БДХПВ характеристик спектральных максимумов, выделенных в результате анализа пространственных спектров 126-и фрагментов космического оптического панхроматического изображения морской поверхности, полученного со спутника Ресурс-П 16.04.2017 в районе п. Кацивели (Крым): а) - распределение значений характеристик спектральных максимумов в координатной плоскости «пространственный период / направление распространения волн»; б) - гистограмма пространственных периодов спектральных максимумов; в) карта спектров различных классов, характеризующихся различными сочетаниями измеренных характеристик спектральных максимумов

Анализ данных, приведённых на рис.3, свидетельствует о наличии на исследуемом участке акватории нескольких систем ветровых волн со средними пространственными периодами $\sim 9$ м и $\sim 29$ м (см. рис.3б). Исходя из рис.3а (распределение значений характеристик спектральных максимумов в координатной плоскости «пространственный период / направление распространения волн») можно провести разделение систем волн не только по длинам, но и по направлениям распространения. Это позволяет выделить в исследуемой акватории как минимум три системы волн: две с пространственными периодами $\sim 9$ м, на направлениях $\sim 30^{\circ}$ и $\sim 95^{\circ}$, и одну систему с пространственным периодом $\sim 29$ м со средним направлением распространения 
$\sim 150^{\circ}$. В процессе мониторинга на основании результатов анализа характеристик спектральных максимумов формируются карты спектров различных классов, характеризующихся различными сочетаниями измеренных характеристик (см. пример на рис.3в). Далее комплекты аналитических материалов (как на рис.3 или подобные) исследуются специалистами-дешифровщиками на предмет выявления признаков антропогенных воздействий в характеристиках наблюдаемого поверхностного волнения [2,5,8].

Приведённый пример иллюстрирует целесообразность применения БД для систематизации и углублённого анализа космических изображений морской поверхности и производных информационных продуктов, формируемых на основании специализированных методов обработки данных дистанционного зондирования Земли. Помимо рассмотренной тематической области (мониторинг пространственной структуры поверхностного волнения в интересах исследования антропогенных воздействий на прибрежные акватории, вызванных глубинными стоками) формирование и использование реляционных БД может быть эффективным решением при реализации других направлений обработки космических изображений морской поверхности и суши (например, [10-13]).

\section{Заключение}

Сформирована база данных характеристик пространственной структуры поверхностного волнения на российском шельфе Чёрного моря (БДХПВ), которая позволяет собирать, систематизировать, хранить и выгружать для анализа данные о спектрах волнения в интересах решения задач космического мониторинга антропогенных воздействий на водную среду. В разработанной БДХПВ используется реляционная модель данных.

Исходные данные (спектры космических изображений, контактные спектры волнения, восстановленные по космическим изображениям спектры уклонов и возвышений морской поверхности) сохраняются в БДХПВ с использованием разработанного клиентского приложения. Необходимые пользователям данные могут быть запрошены у БДХПВ через приложение посредством SQL-запросов, а также путём выполнения фиксированных (часто-используемых) запросов, интегрированных в приложение в виде отдельных интерфейсных функций (кнопок). Массив данных, формируемый в рамках работы БДХПВ, хранится в файловой системе на сетевом хранилище с разграничением прав доступа.

В процессе разработки базы данных были выделены и структурированы свыше 100 типов параметров спектров морского волнения, необходимых для исследований антропогенных воздействий, при этом в БДХПВ используется 7 типов таблиц: двумерные пространственные спектры изображений морской поверхности, восстановленные пространственные спектры возвышений и уклонов морской поверхности, угловое распределения энергии в спектре, координаты спектра, одномерные частотные спектры морского волнения, характеристики ветровых максимумов, характеристики аномальных «квазимонохроматических» максимумов.

Приведены примеры статистической обработки выгруженных из БДХПВ характеристик спектральных максимумов, выделенных на основании интерактивного анализа пространственных спектров космического оптического панхроматического изображения морской поверхности, полученного со спутника Ресурс-П 16.04.2017 в районе п. Кацивели (Крым). Приведённый пример иллюстрирует целесообразность применения реляционных баз данных для систематизации и углублённого анализа информации, генерируемой при обработке космических изображений морской поверхности с использованием методов пространственной спектральной обработки. Исследование обнаруживает перспективные возможности эффективного применения баз данных при реализации различных направлений тематической обработки данных дистанционного зондирования Земли.

Работа выполнена при поддержке Минобрнауки России. 


\section{References}

[1] Bondur V, Tsidilina M. Features of Formation of Remote Sensing and Sea truth Databases for The Monitoring of Anthropogenic Impact on Ecosystems of Coastal Water Areas // 31st International Symposium on Remote Sensing of Environment. ISRSE, 2005. P. 192-195

[2] Bondur V.G., Zamshin V.V. Comprehensive Ground-Space Monitoring of Anthropogenic Impact on Russian Black Sea Coastal Water Areas, in book K.V. Anisimov et al. (eds.), Proceedings of the Scientific-Practical Conference "Research and Development - 2016", 2018. P. 625-637. DOI 10.1007/978-3-319-62870-7

[3] Bondur V.G. Aerospace Methods and Technologies for Monitoring Oil and Gas Areas and Facilities // Izvestiya, Atmospheric and Oceanic Physics. 2011. Vol. 47. No. 9. P. 1007-1018. DOI: $10.1134 /$ S0001433811090039

[4] Zeiler M. Modelirovanie nashego mira (Modeling Our World: The ESRI Guide to Geodatabase Concepts) / Translation from English. Mocow: Data+, 2004. 254 p. (In Russian)

[5] Bondur V. Complex Satellite Monitoring of Coastal Water Areas // 31st International Symposium on Remote Sensing of Environment. ISRSE, 2005. 7p.

[6] Bondur V.G., Dulov V.A., Murynin A.B., Yurovsky Yu.Yu. A study of sea-wave spectra in a wide wavelength range from satellite and in-situ data // Izvestia, Atmospheric and Oceanic Physics. 2016. Vol. 52. No. 9. P. 888-903. DOI: $10.1134 / \mathrm{S} 0001433816090097$

[7] Bondur V.G., Zhurbas V.M., Grebenyuk Yu.V. Mathematical Modeling of Turbulent Jets of Deep-Water Sewage Discharge into Coastal Basins // Oceanology. 2006. Vol.46. No.6. P. 757-771. DOI: $10.1134 / \mathrm{S} 0001437006060014$

[8] Bondur V.G. Satellite monitoring and mathematical modelling of deep runoff turbulent jets in coastal water areas // in book Waste Water - Evaluation and Management, ISBN 978-953-307-233-3, InTech, Croatia. 2011. P. 155-180. http:// www.intechopen.com/articles/show/title/satellite-monitoring-and-mathematical-modelling-ofdeep-runoff-turbulent-jets-in-coastal-water-areas

[9] Leckler F., Ardhuin F., Peureux C., Benetazzo A., Bergamasco F., Dulov V. Analysis and interpretation of frequency-wavenumber spectra of young wind waves // Journal of Physical Oceanography. - 2015. - V.45. - № 10 (October 2015). - P.2484-2496, DOI: 10.1175/JPO-D-14-0237.1

[10] Bondur V.G., Keeler R.N., Starchenkov S.A., Rybakova N.I. Monitoring zagryazneniy pribrezhnykh akvatoriy $\mathrm{s}$ ispolzovaniem mnogospektral'nykh sputnikovykh izobrazheniy vysokogo prostranstvennogo razresheniya (Monitoring of the pollution of the ocean coastal water areas using space multispectral high resolution imagery) // Issledovanie Zemli iz kosmosa. 2006. No. 6. P. $42-49$ (In Russian)

[11] Bondur V.G., Zubkov E.V. Vydelenie melkomasshtabnykh neodnorodnostey opticheskikh kharakteristik verkhnego sloya okeana po mnogozonalnym sputnikovym izobrazheniyam vysokogo razresheniya. Chast 1. Effekty sbrosa drenazhnykh kanalov v pribrezhnye akvatorii (Showing up the small-scale ocean upper layer optical inhomogeneities by the multispectral space images with the high surface resolution. Part 1 . The canals and channels drainage effects at the coastal zone) // Issledovaniya Zemli iz kosmosa. 2005. No. 4. P. 54-61. (In Russian)

[12] Bondur V.G., Sharkov E.A. Statisticheskie kharakteristiki pennykh obrazovaniy na vzvolnovannoy morskoy poverkhnosti (Statistical characteristics of foam formations on a disturbed sea surface)// Oceanology. 1982. Vol.29. No. 3. P. 372-379. (In Russian)

[13] Ivanov A.Yu., Filimonova N.A., Kucheiko A.Yu., Evtushenko N.V., Terleeva N.V. Oil spills in the Barents Sea based on satellite monitoring using SAR: spatial distribution and main sources // International Journal of Remote Sensing, 2017. doi:10.1080/01431161.2017.1371869 